\title{
Verification and Validation in Computational Mechanics
}

\author{
Shuvodeep De \\ Virginia Polytechnic Institute and State University, Blacksburg, VA, 24061-0203
}

Keywords: Verification and Validation, Computational Mechanics, Structural Mechanics, Structural Health Monitoring, Finite Element Model, artificial intelligence, Machine Learning, Multidisciplinary Design Optimization, Complex structures, Topology Optimization, Stress Verification, Simulation, Surrogate Model

Decades ago, when computational was expensive and limited, the structural design was mostly performed by hand calculations using simple mathematical models. For example, it was a common practice to design a structure as complex as the wing of an aircraft by simple beam analysis. However, ever since the classic paper by Turner et al., due to a rapid increase in computational, more complex mathematical models are being used to simulate the physical behavior of complex structural components. To solve intractable problems unsolvable by hand calculations, numerical techniques like Finite Element Analysis (FEA), Computational Fluid Dynamics (CFD), Finite Difference Method etc. are being employed. In fact, the availability of these methods has led to the development of an entirely new area of research known as Multidisciplinary Design Optimization (MDO) where various disciplines are considered in an optimization problem. The most important question while using a mathematical model to represent practical industrial problems is to what extent these models represent the real-life situation. Computational models are always built on upon assumptions. Simply at looking at the simulation outcomes i.e. the graphical and numerical results, it is often very difficult to ensure if the underlying assumption holds and that the results are reliable. This has led to the development of another field of research known as Verification and Validation (called V\&V in short).

\section{Assumptions in Computational Models}

Verification and Validation is a very important and essential field where the computational models[1-14] are tested for their credibility. Computer simulations are used to predict real-life outcomes, control vehicles precisely, design light-weight structures etc. Additionally, they make it possible to predict incidents for which setting up experiments can be infeasible, dangerous or costly. Examples of such incidents include the impact of blast-waves on a ship or submarine structure, consequences of detonating a nuclear warhead on an asteroid etc. In fact, for highly 
complex structures like submarines and ships, the US Navy has become increasingly reliant on computational models and simulations. Many important decisions are made by the engineers designing vehicles for the navy using computational simulation results but they often struggle due to inconsistency in results caused by the use of different computational models. .As mentioned earlier, computational models are developed based on some assumptions. The following assumptions are common while solving problems involving complex structures:

1) For metallic structures, the material is often assumed to be linearly isotropic. This means that material even in the presence of complex microstructure of the metal with grain boundaries, dislocations, voids, micro-cracks etc, is modeled as a homogeneous continuum. The properties of a mechanical component after manufacturing are almost never the same as those predicted by simulations.

2) In reality, the structures are subjected to highly complex and often random loads. In structural design and optimization, the analysis is usually run considering certain idealistic loading conditions.

3) It is a common practice to use idealistic boundary conditions in mathematical models [15-24]. Example of such boundary conditions includes simply-supported, pinned, fixed etc. In reality the boundary is often too complex to be represented by a pure form of any of the above mentioned, idealistic boundary conditions.

4) Many details are excluded at the conceptual level to save computational resources. For example, in the mathematical model of a submarine, the bulkhead is often approximated as a simple plate with T-joints whereas in reality it is a complex structure made of several components. Moreover, in a structural assembly different components are connected using bolted joints, welded joints, rivets etc. The detailed features of these joints are often excluded from the mathematical model. Approximation should be made such that a mathematical model is sufficient to answer the requisite quantitative questions and it requires sound engineering judgement. The major goal in Verification and Validation is to systematically collect evidence to ensure that the computational model has sufficient fidelity for the analyzing engineering component.

\section{Verification and Validation Definitions}


The definition of Validation according to ASME V\&V 10-2006 [25] is "the process of determining the degree to which a model is an accurate representation of the real world from the perspective of the intended uses of the model." Such determination can be made using either data from physical tests (classic validation) or from simulation models with higher fidelity physics (hierarchical validation).

Definition of Verification as quoted from the ASME V\&V 10-2006, is "the process of determining that a computational model accurately represents the underlying mathematical model and its solutions". It is important that the accuracy criteria is specified prior to the initiation of model development and experimental activities for validation purposes. For experimental validation, the $\mathrm{V} \& \mathrm{~V}$ plan should be prepared that will include a detailed specification of the use of the model, description of the global system and its subsystems, their interactions and the list of experiments that needed to be performed. It may also include details about the approach to verify the model.

\section{Hierarchical Model development and Bottom-up approach}

Complex models are usually hierarchical in structure. They consist of components linked together to form subassemblies. Similarly subassemblies form assemblies which again make up the global structure. For V\&V purpose, a bottom-up approach is recommended. The accuracy of each individual component should be validated first followed by their mutual interaction in the subassembly where they belong to and so on. If validation of the system as a whole is done from experimental data, it might lead to the following issues:

1) For complex systems, if there is a significant disagreement between experiment and simulation it often becomes difficult if not impossible to find which of the components are responsible for the disagreement.

2) Even if there is good agreement between the experiment and its simulation, it might be due to error cancellation from different components.

A bottom-up approach will result in confidence in each level of the hierarchical structure.

\section{Conceptual, Mathematical and Computational Model}


The modeling activity starts with a preliminary conceptual model which can be defined as an idealized mechanics-based representation of the reality of interest. It always starts by a set of assumptions about material properties, geometry, interface, boundary conditions and careful engineering judgements are needed so that the model is adequate to simulate reality. Constructing the Phenomena Identification and Ranking Table (PIRT) can be useful for identifying the key physical processes. Qualitative judgement regarding existing computational models might be useful as well for this purpose.

After conceptualization, the next step is to formulate governing mathematical equations for the involving mechanics, interface properties, loads and boundary conditions. It includes specification of model input parameters by the user. Most complex engineering problems are governed by differential equations for which no generalized analytical solution available. The equations are solved using numerical techniques like finite-element methods, finite-difference methods etc. The numerical implementation of the mathematical model is known as computational modelling. It should include the type and degree of special discretization (element type, element size etc), temporal discretization, solution algorithm (like implicit or explicit solver). It is very important to decide the right fidelity for the computational model for the problem to be solved. Problems involving structural optimization are computationally intensive and often requires a large number of analyze. To meet constraints on project time, appropriate fidelity of computational models and parallel computation are extremely helpful. 


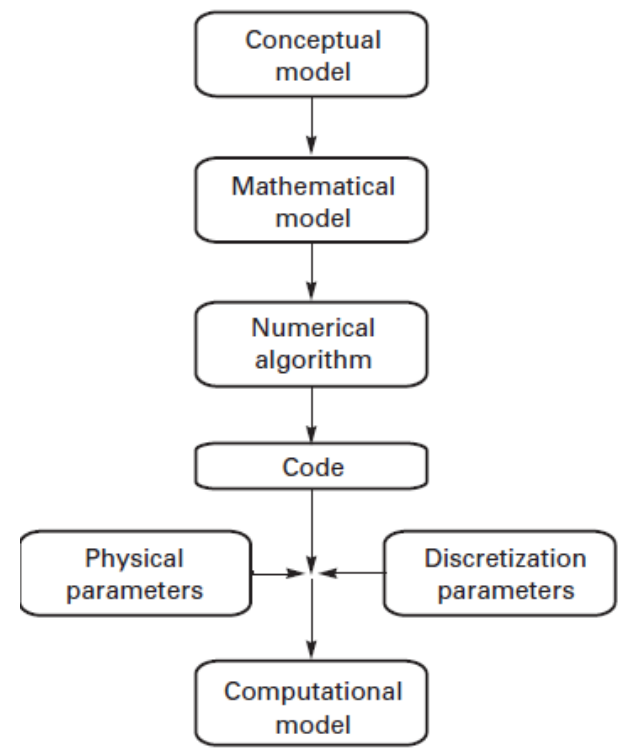

Figure 1: Process of Model Development (Source: ASME V\&V 10-2006)

\section{Metrics for Verification and Validation}

The solution output as a function of the user-provided input parameters is known as the response. The key responses in a computational mechanics problem are as follows:

- Displacements: Displacement fields are easy to measure using Digital Image Correlation (DIC). Displacement can be translational or rotational.

- Energy: Strain energy is the energy stored in the system due to deformation and computed by integration of stress over the strain domain. Depending on the geometry of the component and the loading condition some of the components from the energy expression can be ignored. For example, for a beam subjected to pure bending loads, the strain energy is mostly due to the bending moment and it is called bending strain energy. The total energy input from applied load deflection (which leads to frictional, plastic or viscous dissipation). Energy can be classified as:

- Normal and shear components

- Bending and membrane

- Volumetric and distortional component

○ Principal or other invariant 
- Stress and strain: Stress and strains are most commonly used metrics for V\&V. Currently, there are few techniques available to measure stress in a component directly. For example, using X-ray techniques can be used to estimate stress based on distortion in the crystal lattice, however it is restricted to the outer layer of 5-15 micron depth. Stress is usually expressed in terms of strain data using Hooke's law. The ease of measurement of strain using strain gauges makes it a widely used metric for $\mathrm{V} \& \mathrm{~V}$.

Based on origin and direction, stress and strain can be classified as:

- Normal and shear components

- Bending and membrane

○ Volumetric and distortional component

○ Principal or other invariant

Forces: Forces can be a pointwise component (e.g., reaction force for boundary condition) or sectional component (e.g., local shear force/bending moment resultants).

- Modes: Modes are very important metrics to verify mass and stiffness distribution in a mechanical component. Modes can be static or dynamic (including rigid body modes). Modes can also be determined easily using accelerometers and strain gauges.

Responses are used to formulate metrics for $\mathrm{V} \& \mathrm{~V}$ comparison purposes. Depending on the problem, the following types of metrics are useful:

- Pointwise quantities: It is the response at a particular point

- Pointwise derivatives: It is response variation at a particular location. For example, the degree of stress jump when that stress component should be smoothly varying along a particular path

- Thresholding/Max/min $\left(\mathbf{L}_{\infty}\right)$ norms: It can be calculated from the response over entire structure or over a certain domain of interest.

- Average values: Usually response average over a certain area is used. Average value of response over entire structure is rarely useful.

- Total Energy Sums: It can be computed over entire structure or over regions of interest.

\section{Model Updating}


During the modeling process, the conceptual, mathematical and computational model is usually updated using experimental data to achieve the desired accuracy. This process is commonly known as 'model updating'. Model updating involves calibrating parameters in the mathematical and computational models as well as updating model form. A model calibrated to certain experimental data however may not lead to accurate predictions for all of its uses. Moreover, experimental results are affected by error due to scaling, boundary effects, instrumental resolution and a human factor which introduces additional challenges in the model calibration.

Updating the form of the conceptual mode is observed during quantitative comparison when some structural response is not consistent with corresponding characteristics of the model output and it is found that the difference is not due to inaccurate values of model parameters. One of the most common deficiencies in model form arises when two dimensional models are used for three dimensional structures.

\section{Sensitivity Analysis}

Sensitivity analysis is the process by which the influence of various model input parameters is determined usually by analysis of variance (ANOVA). Sensitivity analysis must be performed after the model is validated. Sensitivity analysis can be local and global. Local sensitivity analysis is used to determine the influence of input parameters on the response in the vicinity of a point. For this purpose adjoint method or finite difference methods are mostly used. In the global sensitivity analysis, the average of the response over a larger domain is calculated.

\section{Uncertainty Quantification}

As part of the validation the uncertainties associated with both simulation and experiment should be identified and reported. Uncertainties are of two types: aleatory and epistemic. Aleatory uncertainties occur due to inherent variability in geometry, material properties, loading condition etc. and they cannot be reduced but they should be quantified by determining the mean value standard deviation etc. Using methods like Latin Hypercube and Monte Carlo simulation, the inherent variability is propagated to determine the expected variability in the simulated outcome. Epistemic uncertainties occur due to lack of proper knowledge about the system. They are again of two types: statistical uncertainties occurring due to use of a limited number of samples and model form uncertainties which occurs due to assumptions about model parameters. 


\section{Verification Procedure}

Verification consists of two fundamental activities: code verification and calculation verification. Code verification ensures there are no programming errors and the numerical algorithms generate accurate solutions. If there are bug reportings, the causes of the bugs are needed to be discovered and removed and this process is known as debugging. Solution Quality Assurance (SQA) methods are used to address configuration management, version control, code architecture, documentation and regression testing.

Solution verification is the process to address sufficient geometric following de-featuring, adequate representation of boundary conditions and applied loads, the correctness of input parameters, the temporal and spatial discretization error, iterative convergence rates, independence of numerical solution to coordinate systems, round-off error. A number of solution verification techniques are currently available including:

- Tribal knowledge of best practices (e.g. Shipyard Specification Package U.S.S Clamagore SS-343 summarizes information on primary submarine components and their stiffness/strength issues)

- Representative problems to verify model generation technique and code solution specification (e.g., Shell to Solid Coupling in panel bending) .This also helps with bug reports.

- Check FEA code warning and error messages

- Free body diagram cuts through the model to verify boundary conditions, contact, connector specification

- Rigid body mode confirmation to verify boundary conditions, contact, connector specification

- Linearity thresholding (e.g., small strains/rotations, stress against material yield/ultimate limits)

- Hand calculation checks

- Total mass / cg location

- Mesh convergence study

- Mesh checks including aspect ratios and free edges 
○ Peak values, paying attention to limits of linear modeling (e.g., corners)

○ Stress spatial variation (e.g., contour smoothness/jumps)

- Energy balance totals (e.g., physical energy vs. artificial energies associated with numerical solution)

- Conservative bounding (e.g., the model set-up that bounds worst case response range)

- Error estimates (if available)

\section{Mesh Convergence Study}

The rate of convergence is estimated by comparing results obtained by different discretization preferably to an exact solution. The difference in response due to different discretization occurs due to various factors like:

- Mesh size and local refinement in simulation (static, quasi-static, dynamic, modal extraction etc.)

- Time step size in quasi-static and dynamic simulations. Example: the quasi-static system is linear viscoelasticity where material has time dependent response regardless of whether the inertia term is important or not (i.e., dynamics is important or not)

- Number of modes used in modal superposition

The solution is said to have achieved convergence if it asymptotically reaches the exact solution as discretization is refined. The rate of convergence is determined by comparing the solution for different discretization to the exact (preferably analytical) solution. However, the analytical solution to a physical problem is known only in a few cases. The method of manufactured solution (MMS) is often used to derive a special type of analytical solution. The level of confidence (high to low) in the solution to which the numerical solution is compared is $[1]$ :

- Exact analytical solution (including manufactured solutions)

- Semi-analytic solutions (reduction to numerical integration of ordinary differential equations 
- Highly accurate numerical solution to PDEs

\section{A Posteriori Error Estimation}

The stresses at the Gauss points in a standard Finite Element Model are known to be superconvergent. Usually, extrapolation of Gauss point stresses to the nodes and then averaging them over the nodes is the standard procedure used to obtain nodal stresses which may be inaccurate. However, by using a posteriori error estimator, a better estimate of the nodal stresses i.e. smoothed stresses can be obtained. The error in smoothed stresses and the stresses estimated by the FEM procedure in the energy norm can be further used for refinement purposes, an essential procedure in FEM, by using $h$ or $p$ refinement. It was shown by Zeinkeiwicz-Zhu[26] that if the recovered derivatives are superconvergent, then the estimator will be asymptotically exact (see Zienkeiwicz-Zhu[27]). The smoothed stresses are generally calculated by fitting the shape functions through these superconvergent integration points resulting in better values at the nodes and throughout the element. Also it is intuitive to have continuous stresses rather than a discontinuous discrete direct evaluation of stresses Oden[28] through linear finite elements like for instance taking the case of the axial bar as presented later in this report.

A posteriori error estimator, to increase the reliability of finite element solution procedure, was developed by Zeinkeiwicz-Zhu [26] based on their smoothed stress solution. After evaluation of the stresses based on a finite element solution, a better smoothed stress solution is obtained to compare and estimating errors resulting from the finite element solution. Ainsworth et al [29] proved the convergence and efficiency of this method. Later, Zienkiewicz-Zhu[26] presented an analogous approach for a local refinement using patch insertion and higher order polynomials. Kelly et al [30] had previously mentioned a posteriori error approach and its application to finite elements, adaptive re-meshing techniques and techniques related to p-convergent methods.

The stresses at integration points are known to be very accurate. The extrapolation of the stress at the integration points are then defined by a linear combination of coefficients and the displacement shape function. A different approach uses an insertion patch[26] (i.e. higher order polynomial in a local region) in linear combination to the coefficients. The coefficients for these shape function or polynomials are found such that they match the nodal stresses. The smoothed stresses can be used to calculate relative errors and further used for refinement using fewer trials. 
The work is applicable to any linear finite element-based discretization. These improved stresses at the nodes give accurate values as compared to nodal averaging which were used traditionally.

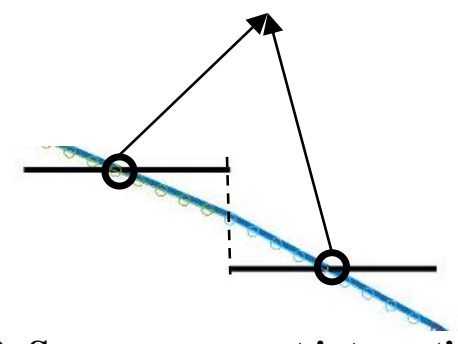

Figure 2: Superconvergent integration points

Example: The process is demonstrated using a problem involving an axial bar with uniformly varying load and a point load at the free end. Figure 3 shows the displacement profile for the axial bar as obtained using the analytical method and finite element method (with 4 elements). Figure 4 shows that stress as computed from FEM is discontinuous and it is compared with smoothed stress.

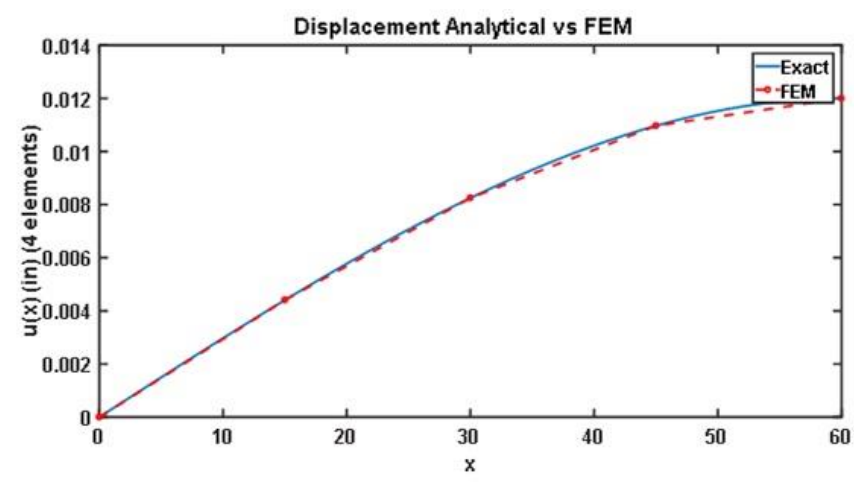

Figure 3: Displacement profile (Analytical vs FEM) for an axial bar with uniformly varying load and end load

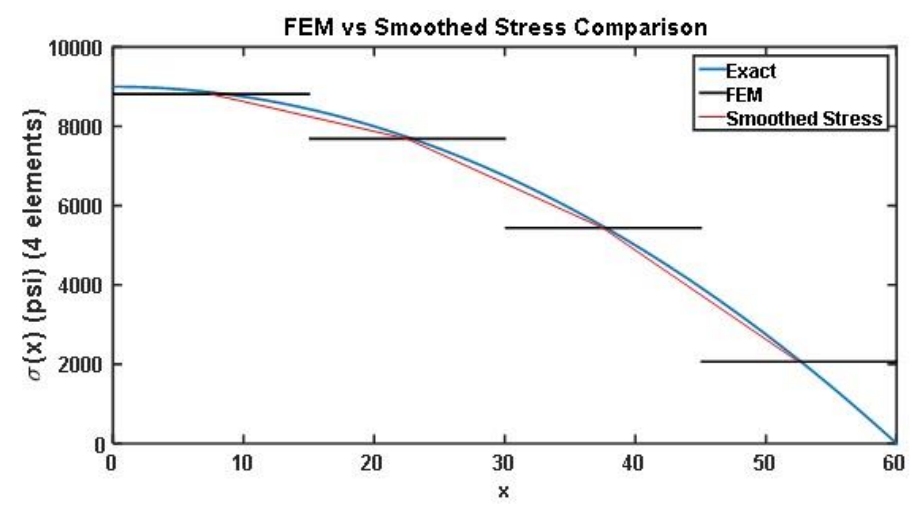

Figure 4: FEM vs Smooth Stress comparison for axial bar with uniformly varying load and end point load 
A similar approach can be employed to 2D (quad, tria, etc.) or 3D (hex, tetra, etc.) elements. Instead of interpolation of the smoothed stresses using shape function in another approach uses a higher order set of polynomial as an interpolation function. The critical areas under consideration, where stress values are needed to be improved or refined, can be defined by patches, to evaluate smooth stresses in that patch. Usually a patch consists of all the elements shared by the node under consideration where stresses are required to be improved.

\section{Classical Validation}

Classical validation is comparing simulation results to physical test data. The modeler and the experimentalist usually work in parallel. The experimental error and uncertainties needed to be tracked. After the mathematical model is formulated the modeler spends some time to ensure there is no bug in the code i.e. for 'code verification' and then finalizes the computational model. Usually for code verification the modeler uses a much simple geometry, boundary conditions and loads than the actual validation problem. The next step taken by the modeler is 'calculation verification' where the purpose is to quantify numerical precision and discretization accuracy for calculations obtained using the computational model. The preliminary calculations using the computational model including sensitivity analysis and uncertainty estimation are often very useful for the design of validation experiment. For example, these data can be used to decide the location of the sensors and the type of measurements that are important. Once the simulation results and experimental results are obtained, they are compared quantitatively. Agreement between the results is decided based on certain criteria which take into account the uncertainties associated with the results. If the agreement is not achieved the conceptual model is updated and the process continues in an iterative way. The process as described is implemented in every level of the hierarchical structural model with a bottom-up approach. Figure 5 shows the entire process of classical verification and validation. 


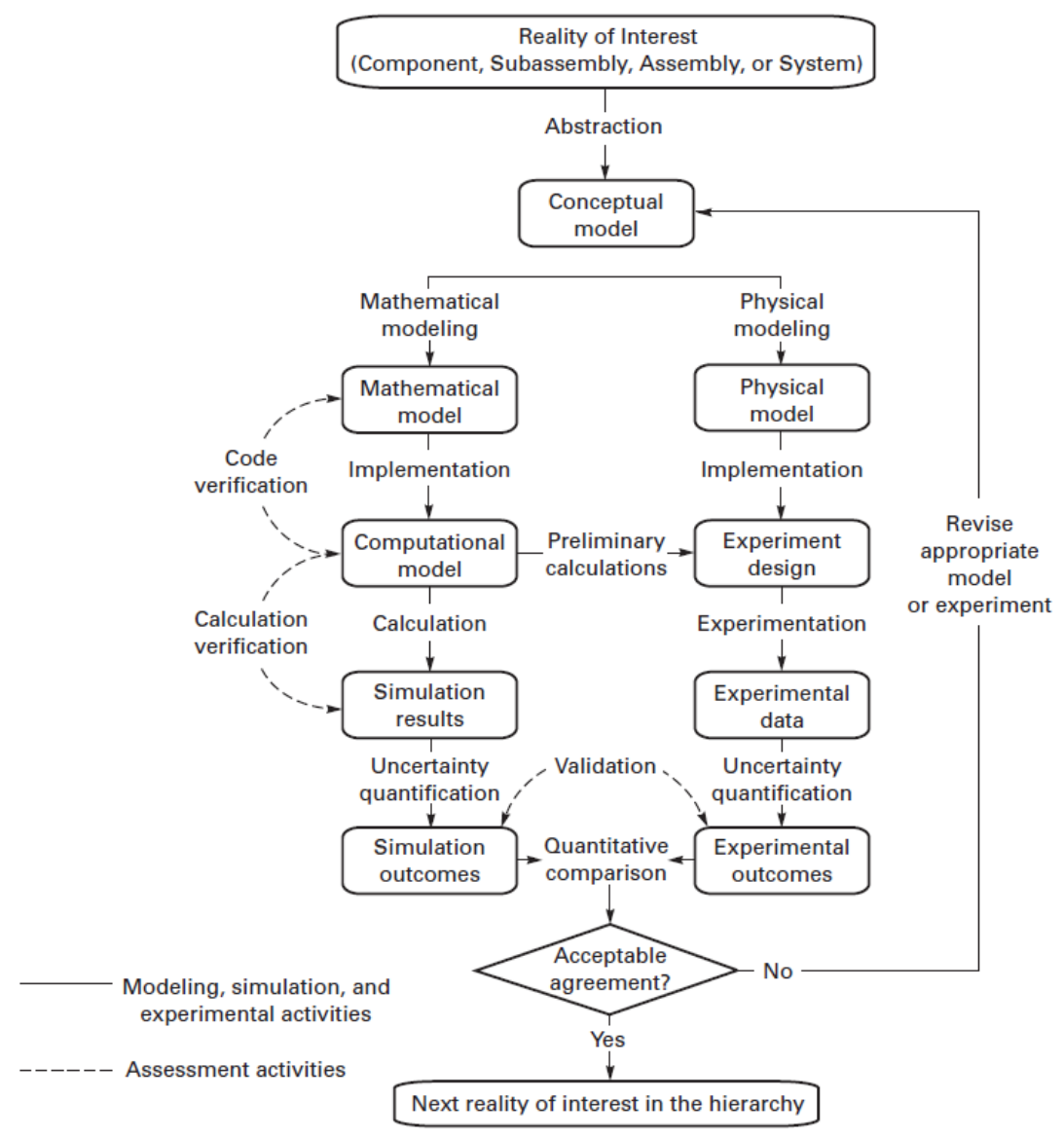

Figure 5: Classical Verification and Validation by comparing simulation outcomes with the experimental outcome (Source: ASME V\&V 10-2006 [1])

The validation needs to be achieved by acquiring data from specially designed experiments. The process should be standardized to build credibility among clients and customers. For computational solid mechanics problems, the strain is one of the most popular metrics used for validation since strain is easy to measure using strain-gauges. It is a common practice to attach strain gauges at regions of high stress however this method of traditional validation has a number of drawbacks:

1) The location of high stress is determined by guess or by results of the computational model. Thus, the approach is somewhat a circular process. There is always a chance that failure can initiate somewhere else.

2) The regions of low stress are vastly ignored in the validation process. It is a common practice to remove materials from these regions (example: by making holes and cutouts on 
the ribs of an aircraft wing). Since validation is not performed for these regions, it results in an unquantified risk of component failure.

3) A point to point comparison of strain-data obtained from different sources is computationally expensive. The strain data are usually obtained from photo-elasticity, thermoselastic analysis or digital field correlation and the number of points has an order of magnitude of $10^{6}$ or greater. Thus the number of operations for point-to-point comparison can have an order of magnitude as high as $10^{12}$. On top of that, data acquired from two different sources might have a different coordinate system, scales etc. which makes the comparison even more expensive.

Sebastian et al [31] discussed an alternate but useful method of validation using image processing. In this approach the strain field obtained experimentally and that obtained by finite element analysis are considered to be analogous to digital images where the value of strain is represented by gray level or color values. Each of the images is decomposed into shape descriptors which are a true representation of features in the corresponding images. The shape descriptors need to be invariant under translation, rotation and change of scale and thus preserve the information in the images. Shape descriptors include wavelet descriptors, Fourier descriptors and orthogonal geometric moments such as Zernike moments, Tchebichef moments, Krawtchouk moments etc. Zernike moments are based on polar coordinates and work best for images with rotational symmetry. Tchebichef and Krawtchouk are based on Cartesian coordinate and are discrete and thus they are easier to be used for most strain data. Figure 6 shows the validation framework using image processing. The accuracy of the feature vectors is ensured by reconstructing the original image using the feature vector and computing the average squared residual. The average residual should not be greater than the minimum measurement uncertainty. If the reconstructed image does not fit well with the original image an alternate method of image decomposition is chosen or the present method is refined. Once the correct feature vectors are found, the feature vectors of the simulation results $(\mathrm{SM})$ and the experiment results (SE) are compared. Validation of the computation is achieved when for all points: 


$$
s_{M}=s_{E} \pm u_{E}
$$

where, $\mathrm{u}$ is the uncertainty of data from an experiment.

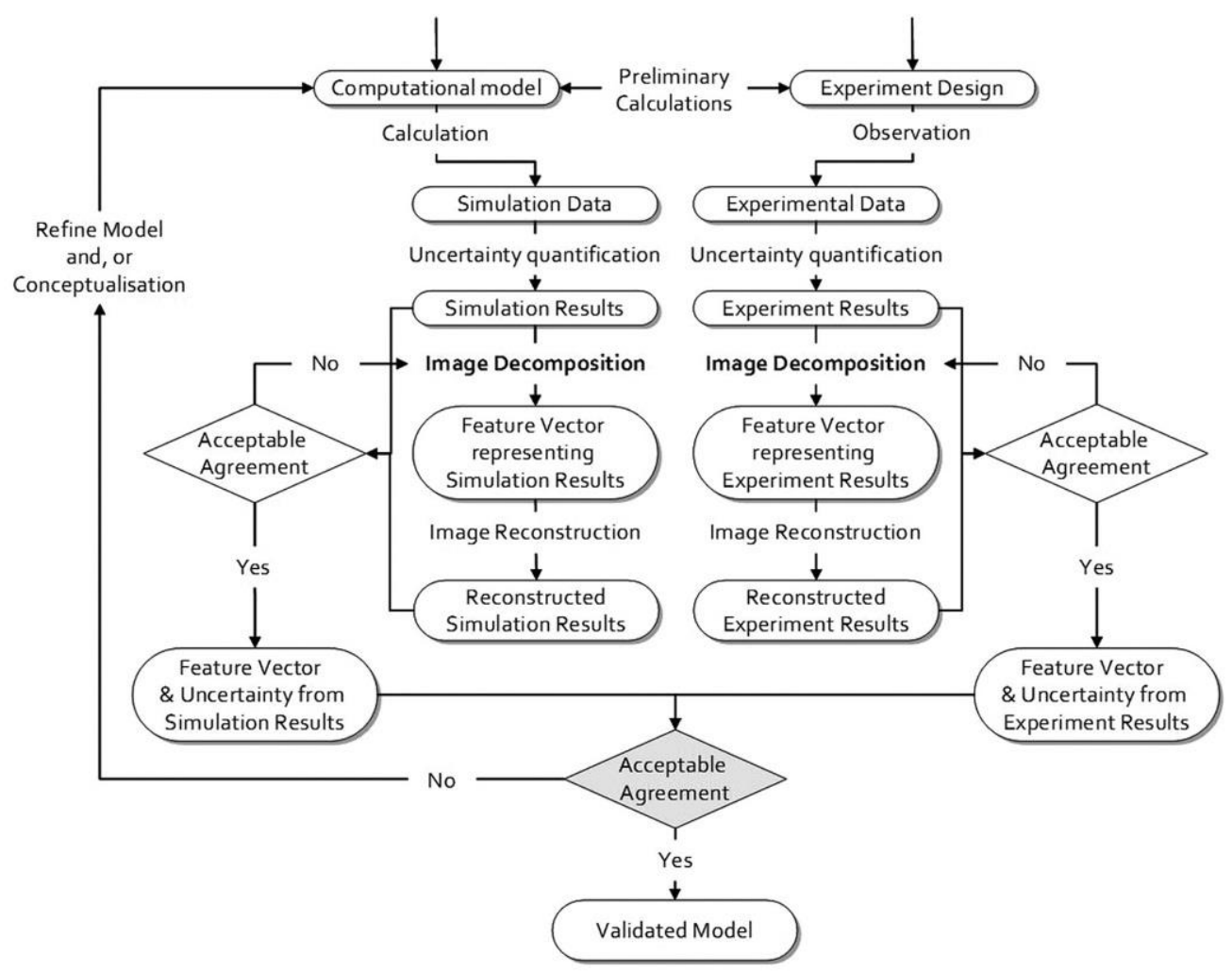

Figure 6: Validation by image processing (Source:[8])

\section{Hierarchical Validation}

Even though according to the strict definition of validation, simulation results must be compared to experimental data, the latter is not often available. In this work, an alternative method of validation is proposed where the comparison is made with a higher fidelity model instead of experimental data. The term 'hierarchal validation' is used to denote the concept of a family of mathematical models of increasing physics fidelity. Such higher fidelity physics models themselves will lead to higher fidelity computational models which again are subject to verification and validation. Hence, ultimately all models must be subjected to classical validation, but in the absence of available physical test data, hierarchical validation can be used to provide significant confidence in modeling results. The hierarchical validation concept can be best illustrated by example. For instance, the results from a small strain/small rotation, linear elastic, Euler-Bernoulli beam model can be compared against those from a large-strain/large-rotation elastic-plastic 3-D continuum response of the same beam geometry. If the result quantities of interest for the intended 
use are similar for representative beam geometries and loads in the intended application, then the small strain/small rotation, linear elastic, Euler-Bernoulli beam model can be used with confidence until such time when comparison with experimental data is necessary (e.g., certification). The process of hierarchical validation is shown in Figure 7.

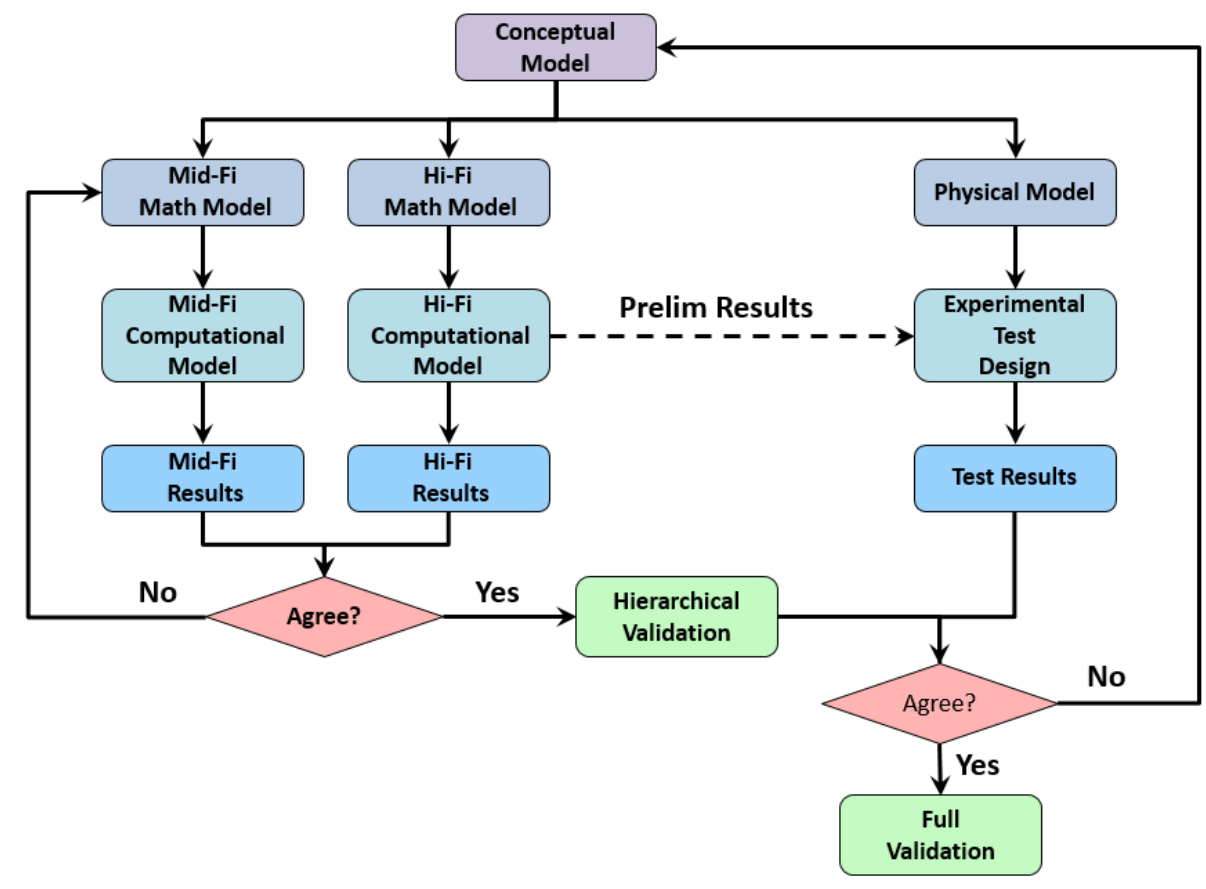

Figure 7: Hierarchal Validation

In the following section, the comparison of lower fidelity to higher fidelity is explained using a few examples:

- Geometry/domain representation: To save computational cost, the modeler often develops a simplified model of the component. Often details like small holes, and fillets are ignored. Such a model of low fidelity can be compared with a model with all geometric features which serves as a high-fidelity model.

- Material physics: Assuming linearity in material physics is an idealization and in the real world nothing is strictly linear. Model with non-linear material physics can be considered as a high- fidelity model with which linear models can be compared. For example a linear elastic model can be compared with one which takes into account elastic-plastic response with kinematic or isotropic hardening. Similarly, a homogenized orthotropic fiber matrix is a low-fidelity representation of the material physics which can be validated against a model where all fibers are explicitly modeled. 
- Strain-displacement relations: Popular beam equations assume what form the structural response will have a priori. For example, Euler-Bernoulli assumes plane sections remain plane and plane sections normal to the neutral axis remain neutral to the neutral axis. Such assumptions correspond to assuming a structural strain-displacement relation a priori relative to the classic strain-displacement equations for 3-D continuum. A model with Euler-Bernoulli assumption can be validated against high-fidelity models like 3D beam or Timoshenko beam (where, plane sections remain plane, but it can rotate about the neutral axis as appropriate for short/thick beams). The Kirchoff-Love plate is the plate equivalent of Euler-Bernoulli beam and it can be verified against 3D continuum model or Mindlin shell (which allows transverse shear for thick shells)

- Contact representation: Contact between two components usually have a complex mechanics and is represented by a number of simple models like fixed contact and sliding contact. These representations can be validated against more complex models like full contact.

- Finite Element Models: Components with thin geometry are often meshed using 2D shell elements which can be validated against full 3D models.

\section{Solid vs Shell Comparison}

Finite element analysts face a lot of challenges to create the appropriate mesh for the problem being solved. Shell elements may be considered as mathematically simplified solid elements [10]. Shell elements are of two types: thin shell elements and thick shell element. Thin shell elements do not consider stress in the direction perpendicular to its surface as thick shell elements consider stress in direction to the middle surface and take into account shear deformation. The industry is often found to trust more simulations performed with solid-mesh, however there is always a concern about computational resources and also issues like hourglass modes and membrane and shear locking. For thin-walled geometries like the fuselage of aircraft, airfoil skin of aircraft wings, submarine hulls etc. using shell elements over solid element can provide a lot of advantages including significant reduction of computational time. Some of the advantages are as follows: 
1) The difference in stress, strain and displacement vary significantly for solid mesh often vary significantly especially in problems with high magnitude of forces and moments. When the mesh is coarse this difference is more pronounced. When the mesh is refined, the difference becomes less but still a lot of iterations are required to achieve mesh convergence.

2) In order to include all details of the model the edges of an element should be smaller than the smallest detail of the geometry. Sometimes a detailed solid CAD model can have very small edges and while attempting to generate the mesh using any commercial FEA software, it often fails. In order to generate the solid mesh the industry spends significant resources to remove features from the solid CAD model. In such situations it often becomes advantageous to define the mid-surface model for the original geometry and use shell elements for the FEM.

3) In order to generate solid FEM for geometries with thin features, at least 3-4 elements across the thickness is needed to capture all bending and stiffness effects for the accurate solution. For complex parts like injection molded parts with stiffeners, draft angles, fillets etc. the necessary number of elements is often huge, resulting in large computational time.

4) The post-processing time for solid models with a large number of elements is also very long. This becomes an issue when a large number of plots for visualization generated.

5) When shell elements are used, the wall thickness is captured as a mathematical value instead of accurately modeling the thickness. This results in a lesser number of equations to be solved. Since the shell approach is significantly less computationally intensive than the solid approach, it has a huge advantage in problems with iterative simulations.

6) While using solid elements for extremely thin geometry there is a much higher chance that the elements can get inverted causing the so called negative Jacobean error. This do not occur when shell elements are used.

Most of the finite element analyst develops a hybrid model consisting of beam, shell and solid elements. For geometries with thin features, a choice of shell or solid element is usually made 
based on the analyst's previous knowledge. The choice is subjective and often depends on the experience of the analyst and thus there is a possibility of difference in simulation outcome as a result of different finite element models.

\section{Verification and Validation in Sub-project}

Verification and Validation have multiple uses in the current project including solving representative numerical problems, test plan development and design guide documentation. The key results for a model with $100 \%$ 3D elements in compared with multi-fidelity models (comprising of both 2D and 3D elements). Maximum von Mises Stress, maximum principal stress, maximum principal strain, maximum displacement and total strain energy are chosen as metrics. Simple thresholding to this response using Artificial Neural Network (ANN) lead to awareness about right choice among the model hierarchy.

\section{References}

1. Jrad, M., De, S. and Kapania, R.K., 2017. Global-Local Aeroelastic Optimization of Internal Structure of Transport Aircraft Wing. In 18th AIAA/ISSMO Multidisciplinary Analysis and Optimization Conference (p. 4321).

2. De, S., Jrad, M. and Kapania, R.K., 2019. Structural optimization of internal structure of aircraft wings with curvilinear spars and ribs. Journal of Aircraft, 56(2), pp.707-718.

3. Robinson, J.H., Doyle, S., Ogawa, G., Baker, M., De, S., Jrad, M. and Kapania, R.K., 2016. Aeroelastic Optimization of Wing Structure Using Curvilinear Spars and Ribs (SpaRibs). In 17th AIAA/ISSMO Multidisciplinary Analysis and Optimization Conference (p. 3994).

4. De, S., White, J., Brusuelas, T., Patton, C., Koh, A. and Huang, Q., 2020. Electrochemical behavior of protons and cupric ions in water in salt electrolytes with alkaline metal chloride. Electrochimica Acta, 338, p.135852.

5. De, S., Jrad, M., Locatelli, D., Kapania, R.K. and Baker, M., 2017. SpaRibs geometry parameterization for wings with multiple sections using single design space. In 58th AIAA/ASCE/AHS/ASC Structures, Structural Dynamics, and Materials Conference (p. 0570).

6. Biswas, S., Saha, D., De, S., Cobb, A.D., Das, S. and Jalaian, B.A., 2021, June. Improving differential evolution through bayesian hyperparameter optimization. In 2021 IEEE Congress on Evolutionary Computation (CEC) (pp. 832-840). IEEE.

7. De, S., Sides, W.D., Brusuelas, T. and Huang, Q., 2020. Electrodeposition of superconducting rhenium-cobalt alloys from water-in-salt electrolytes. Journal of Electroanalytical Chemistry, 860, p.113889.

8. De, S., Singh, K., Alanbay, B., Kapania, R.K. and Aguero, R., 2018, November. Structural Optimization of Truck Front-Frame Under Multiple Load Cases. In ASME International Mechanical Engineering Congress and Exposition (Vol. 52187, p. V013T05A039). American Society of Mechanical Engineers.

9. De, S., Singh, K., Seo, J., Kapania, R., Aguero, R., Ostergaard, E. and Angelini, N., 2019. Unconventional Truck Chassis Design with Multi-Functional Cross Members (No. 2019-010839). SAE Technical Paper.

10. De, S. and Huang, Q., 2021, May. Mathematical Modeling of Cyclic Voltammogram Curves of Copper Deposition Involving Multiple Additives. In ECS Meeting Abstracts (No. 28, p. 977). IOP Publishing. 
11. De, S., Singh, K., Seo, J., Kapania, R.K., Ostergaard, E., Angelini, N. and Aguero, R., 2021. Lightweight Chassis Design of Hybrid Trucks Considering Multiple Road Conditions and Constraints. World Electric Vehicle Journal, 12(1), p.3.

12. De, S. and Huang, Q., 2020, November. Estimation of Kinetic Parameters of Additives By SemiAnalytical Solution. In ECS Meeting Abstracts (No. 17, p. 1483). IOP Publishing.

13. Huang, Q., Sides, W.D., De, S. and Hu, Y., 2019, September. Electrodeposition of Cu, Co and Re from Water in Salt Electrolytes. In ECS Meeting Abstracts (No. 46, p. 2116). IOP Publishing.

14. De, S., 2017. Structural Modeling and Optimization of Aircraft Wings having Curvilinear Spars and Ribs (SpaRibs) (Doctoral dissertation, Virginia Tech).

15. De, S., Jrad, M., Locatelli, D., Kapania, R.K., Baker, M. and Pak, C.G., 2017. SpaRibs Geometry Parameterization for Wings with Multiple Sections using Single Design (No. AFRC-DAATN37932).

16. Devarajan, B. and Kapania, R.K., 2020. Thermal buckling of curvilinearly stiffened laminated composite plates with cutouts using isogeometric analysis. Composite Structures, 238, p.111881.

17. Miglani, J., Devarajan, B. and Kapania, R.K., 2018. Thermal buckling analysis of periodically supported composite beams using Isogeometric analysis. In 2018 AIAA/ASCE/AHS/ASC Structures, Structural Dynamics, and Materials Conference (p. 1224).

18. Li, Q., Devarajan, B., Zhang, X., Burgos, R., Boroyevich, D. and Raj, P., 2016. Conceptual design and weight optimization of aircraft power systems with high-peak pulsed power loads (No. 2016-011986). SAE Technical Paper.

19. Devarajan, B. and Kapania, R.K., 2022. Analyzing thermal buckling in curvilinearly stiffened composite plates with arbitrary shaped cutouts using isogeometric level set method. Aerospace Science and Technology, p.107350.

20. Devarajan, B., 2019. Thermomechanical and vibration analysis of stiffened unitized structures and threaded fasteners (Doctoral dissertation, Virginia Tech).

21. Miglani, J., Devarajan, B. and Kapania, R.K., 2021. Isogeometric Thermal Buckling and Sensitivity Analysis of Periodically Supported Laminated Composite Beams. AIAA Journal, pp.1-10.

22. Devarajan, B., 2021. Vibration Analysis of Timoshenko Beams using Isogeometric Analysis. arXiv preprint arXiv:2104.12860.

23. Devarajan, B., 2021. Free Vibration analysis of Curvilinearly Stiffened Composite plates with an arbitrarily shaped cutout using Isogeometric Analysis. arXiv preprint arXiv:2104.12856.

24. Devarajan, B., Locatelli, D., Kapania, R.K. and Meritt, R.J., 2016. Thermo-Mechanical Analysis and Design of Threaded Fasteners. In 57th AIAA/ASCE/AHS/ASC Structures, Structural Dynamics, and Materials Conference (p. 0579).

25. Schwer, L.E., 2009. Guide for verification and validation in computational solid mechanics.

26. Zienkiewicz, O.C. and Zhu, J.Z., 1987. A simple error estimator and adaptive procedure for practical engineerng analysis. International journal for numerical methods in engineering, 24(2), pp.337-357.

27. Zienkiewicz, O.C. and Zhu, J.Z., 1992. The superconvergent patch recovery and a posteriori error estimates. Part 1: The recovery technique. International Journal for Numerical Methods in Engineering, 33(7), pp.1331-1364.

28. Oden, J.T. and Brauchli, H.J., 1971. On the calculation of consistent stress distributions in finite element approximations. International Journal for Numerical Methods in Engineering, 3(3), pp.317325.

29. Ainsworth, M., Zhu, J.Z., Craig, A.W. and Zienkiewicz, O.C., 1989. Analysis of the ZienkiewiczZhu a-posteriori error estimator in the finite element method. International Journal for numerical methods in engineering, 28(9), pp.2161-2174.

30. Kelly, D.W., De SR Gago, J.P., Zienkiewicz, O.C. and Babuska, I., 1983. A posteriori error analysis and adaptive processes in the finite element method: Part I- error analysis. International journal for numerical methods in engineering, 19(11), pp.1593-1619.

31. Sebastian, C., Hack, E. and Patterson, E., 2013. An approach to the validation of computational solid mechanics models for strain analysis. The Journal of Strain Analysis for Engineering Design, 48(1), pp.36-47. 